\title{
Nursing Staff's Knowledge and Attitudes toward Dementia: A Pilot Study from an Indian Perspective
}

\author{
Benedicte Sørensen Strøm ${ }^{a} \quad$ Knut Engedal $^{b, c}$ Lasse Andreassen ${ }^{a}$ \\ a Faculty of Health Studies, VID Specialized University, Oslo, Norway; \\ ${ }^{b}$ Norwegian Advisory Unit for Aging and Health, Vestfold Hospital HF, Tønsberg, Norway; \\ 'Department of Geriatrics, Oslo University Hospital, Tønsberg, Norway
}

\section{Keywords}

Dementia $\cdot$ Attitude $\cdot$ Knowledge $\cdot$ India $\cdot$ Nursing

\begin{abstract}
Background: Despite the increased prevalence of dementia in India, reports indicate little awareness of the disease. Symptoms are often misinterpreted or neglected, which could lead to late diagnosis, reducing the choices available regarding future care. Considering that most nurses caring for the elderly will work with people with dementia in the future, there is concern surrounding their ability to meet the needs of these patients, requiring them to obtain the necessary knowledge and positive attitudes for treatment. Aims: To describe the knowledge of and attitudes toward dementia among nursing staff working in residential care facilities for elderly populations in India. Methods: A cross-sectional survey was conducted in 3 nursing homes in India in which 15 nursing staff conducted self-assessments of their knowledge and attitudes toward dementia using the Alzheimer's Disease Knowledge Scale (ADKS) and the Attitude toward Alzheimer's Disease and Related Dementias Scale (DAS) for each respective assessment. Descriptive statistics were used to describe staffs' attitudes and knowledge concerning dementia. Continuous variables were presented as means $\pm S D$, while the categorical variables were presented as percentages. Results: Although the majority of the participants answered that Alzheimer's disease cannot be cured, about half reported that people with dementia, in rare cases, can recover from the disease. While all participants agreed that people with dementia can feel when others are kind to them, almost half disagreed that people living with dementia can enjoy life. Conclusion: These findings reveal that nursing staff have limited knowledge of dementia, but their attitudes toward people living with dementia tend to be positive.




\section{Introduction}

There are an estimated 900 million people worldwide who are 60 years or older, and the elderly population is forecasted to grow. The vast majority of this growth is predicted to come from low- or middle-income countries [1]. The elderly population in India is growing rapidly [2], with the country ranking among the top in the world for the largest population of older people [3]. Increased life expectancies will lead to heightened needs for care and support of the elderly [4]. Furthermore, due to the rapid increase in older people in need of care, a limited time is available to develop services to respond to the ageing population and their needs [5].

In 2015, an estimated 46.8 million people worldwide were living with dementia, and this number is predicted to almost double every 20 years, reaching 74.7 million by 2030 and 131.5 million by 2050 [1]. While dementia is not an inevitable part of normal ageing, it is more common among older people as brain ageing is a major risk factor. The incidence of dementia increases exponentially with age, doubling every 6.3 years, i.e., from 3.9 out of 1,000 persons aged 60-64 years experiencing dementia to 104.8 out of 1,000 persons aged 90 or older having the condition $[1,6]$. Almost $6 \%$ of all people living with dementia live in low- or middle-income countries, with this number expected to increase to $68 \%$ by 2050 [1].

In 2010 , it was estimated that nearly 3.7 million Indians have dementia. This number is estimated to increase $300 \%$ by 2040 [1]. Despite this, dementia as a disease is still not widely recognized in India unless it is fairly advanced [7-9], and it is seldom recognized as an organic brain syndrome or a real medical condition $[1,10]$. Family members often interpret early signs of dementia as signs of normal ageing or as depressive symptoms, often leading to the disease being neglected by both the family and health policy makers [7-9]. Even though it is currently still considered shameful to place parents or relatives in long-term care facilities [11], the Indian family structure is poised to change. More and more older people with dementia in need of care around the clock are being cared for by health care professionals in residential care facilities [1,7]. However, there is a lack of statistics indicating how many people with dementia are living in long-term care facilities [12].

The complexity in caring for people with dementia could be one reason why care staff generally have negative attitudes toward people with dementia [13], and it could also explain why nursing students have reported having negative experiences with these patients in clinical practice [14]. Another reason for students' negative experiences in caring for people with dementia could originate from their nursing teachers' lack of knowledge about the disease [15]. These findings are concerning, as nearly all Indian nursing students are expected to encounter older people with dementia in their work after finishing school [16].

Studies on nursing students' attitudes toward and knowledge on caring for people with dementia have shown that students know next to nothing about how to care for this specific group of patients $[17,18]$. Even though studies have shown that training health care professionals on working with people with dementia can help to effectively deal with behavioral problems and improve health care delivery $[19,20]$, the Dementia India Report [12] states that no special emphasis on the diagnosis and management of dementia exists in training for healthcare professionals in India.

Hence, the aim of this study is to describe the knowledge and attitudes surrounding dementia among nursing staff working in residential care facilities for elderly individuals in India. 


\section{Method}

This study was designed as a cross-sectional survey conducted in 3 nursing homes ( $\mathrm{NH}$ ) in different parts of India, all run by religious orders; of these, 2 (NH A and NH B) care for lay people and 1 cares specifically for members of the order (NH C). All English-speaking nursing staff from the $3 \mathrm{NH}$ were invited to participate. Seven staff from NH A, 4 from $\mathrm{NH} \mathrm{B}$, and an additional 4 from $\mathrm{NH} C$ participated, with nobody declining to participate. In addition to being provided written information about this study, participants were informed about the assessment tools and how to use them, and they were ensured that data would be collected and analyzed anonymously. Participants filled out the assessment tools within 30 minutes. Nine Norwegian nursing students, all fluent in English, administered the assessment and collected the data from January to February 2018.

\section{Assessments}

Alzheimer's Disease Knowledge Scale

Nursing staffs' knowledge of Alzheimer's disease was assessed using the Alzheimer's Disease Knowledge Scale (ADKS) [21]. The scale, which is reported to have good psychometric properties with a high degree of internal consistency (i.e., 0.71), was developed to reflect and quantify the individual's actual knowledge and bias regarding Alzheimer's disease and related disorders [22]. This validated tool consists of 30 items with true/false responses in which 1 mark is awarded for every correct response and zero marks are given for incorrect responses. Hence, the total number of marks achievable in the knowledge section ranges from 0 to 30, with a higher score indicating more knowledge. The ADKS measures knowledge levels within the following 7 different domains of Alzheimer's disease: life impact ( 3 items), risk factors ( 6 items), symptoms ( 4 items), treatment and management (4 items), assessment and diagnosis (4 items), care giving (5 items), and course of the disease (4 items).

Attitude toward Alzheimer's Disease and Related Dementias Scale

The Attitude toward Alzheimer's Disease and Related Dementias Scale (DAS) was used to assess nursing staffs' attitudes about dementia [23]. The DAS reflects the affective, behavioral, and cognitive components of an attitude, and the assessment is reported to have good psychometric properties (Cronbach's $\alpha=0.83-0.85$ ) [23]. The instrument consists of 20 items on a 7-point Likert scale with responses ranging from "strongly disagree" to "strongly agree" to reveal nursing staffs' attitudes toward dementia. The total scores achievable for this scale range from 20 to 140, with a more positive attitude reflected by a higher score. Two subdomains consist of "dementia knowledge" (items 3, 7, 10,11,12, 14, 15, 18, 19, and 20) and "social comfort" (items 1, 2, 4, 5, 6, 8, 9, 13, 16, and 17) [23].

\section{Analysis}

Descriptive statistics were used to identify characteristics of the participants, such as gender, age, work experience with elderly populations, and education level, as well as to describe nursing staffs' attitudes and knowledge surrounding dementia. Categorical variables were presented as percentages, while the continuous variables were presented as means \pm SD. The negative responses were reverse coded. 


\section{Results}

In the present study, 15 English-speaking nursing staff from 3 different $\mathrm{NH}$ in India took part in the survey. The mean age of the nursing staff was $52.6 \pm 17.4$ years, and all of them were women. The average length of work experience in caring for elderly populations was $7.7 \pm 11$ years, with 7 participants $(46.7 \%)$ having over 5 years of experience. In total, 10 $(66.7 \%)$ members of the nursing staff were registered nurses, and $5(33.3 \%)$ were nursing assistants or had no formal education in health care.

\section{Knowledge about Dementia}

The overall mean score for knowledge about dementia was $19.5 \pm 3.0$, with the following scores earned for each of the subcategories: $2.1 \pm 0.9$ for life impact, $2.8 \pm 0.7$ for assessment and diagnosis, $3.2 \pm 1.0$ for caregiving, and $2.2 \pm 1.0$ for course of dementia. While 9 members of the nursing staff $(60 \%)$ agreed that people with dementia are particularly prone to depression, as many as $11(73.3 \%)$ agreed that symptoms of severe depression can be mistaken for symptoms of dementia. Five (33.3\%) indicated that the average life expectancy of a dementia patient is 6-12 years after symptoms appear. Even though as many as 14 (93.3\%) subjects answered that dementia cannot be cured, 8 (53.3\%) answered that people with dementia, in rare cases, can recover from the condition (Table 1).

\section{Attitudes toward Dementia}

The overall mean score for attitudes toward dementia was $107.9 \pm 10.9$ (Table 2). Concerning nursing staff's attitudes toward dementia in the social comfort domain, the mean score of $50.3 \pm 9.2$ showed that the staff had an overall positive attitude toward caring for people with dementia. The majority (93.3\%) felt that it was rewarding working with dementia patients. As many as $13(86.7 \%)$ subjects felt comfortable touching them, while $8(53.3 \%)$ agreed that they were not very familiar with dementia and felt frustrated because they did not know how to help those experiencing it. In NH B, only 1 staff member (25\%) expressed familiarity with dementia, while the remaining 3 members (75\%) felt frustrated due to their lack of knowledge about how to help persons with dementia.

The mean score for the knowledge domain was $57.6 \pm 5.4 ; 14$ (93.3\%) respondents agreed that every person has different needs and that it is important to know an individual's history. In this survey, 11 (73.3\%) respondents agreed that they could do a lot to improve the lives of people with dementia and that residents' challenging behaviors could be a form of communication. However, the majority (75\%) of the staff in NH B did not agree that difficult behaviors could be a form of communication. While all participants in the $3 \mathrm{NH}$ agreed that people with dementia feel when others are kind to them, only $2(50 \%)$ subjects in NH B agreed that they can enjoy life, and 4 (57.1\%) and 3 (75\%) subjects each in NH A and NH C, respectively, agreed with this. Furthermore, all of the participants in $\mathrm{NH} \mathrm{C}$ expressed that it is possible to enjoy interacting with people with dementia. Five (71.4\%) subjects in NH A agreed with this, whereas only 1 (25\%) in NH B agreed on the matter.

\section{Discussion}

The aim of this study is to describe nursing staffs' knowledge of and attitudes toward dementia. The findings reveal that the nursing staffs studied have limited knowledge of dementia. However, the nursing staff did report having positive attitudes toward people living with dementia. 
Table 1. Knowledge related to Alzheimer's disease (ADKS)

\begin{tabular}{|c|c|c|}
\hline & $\begin{array}{l}\text { Correct } \\
\text { answers, \% }\end{array}$ & Mean \pm SD \\
\hline \multicolumn{3}{|l|}{ Life impact } \\
\hline People with Alzheimer's disease are particularly prone to depression (T) & 60 & $0.6 \pm 0.5$ \\
\hline Most people with Alzheimer's disease live in nursing homes (F) & 86.7 & $0.9 \pm 0.4$ \\
\hline $\begin{array}{l}\text { It is safe for people with Alzheimer's disease to drive, as long as they have a companion in } \\
\text { the car at all times (F) }\end{array}$ & 60 & $0.6 \pm 0.5$ \\
\hline Mean score & & $2.1 \pm 0.9$ \\
\hline \multicolumn{3}{|l|}{ Risk factors } \\
\hline \multicolumn{3}{|l|}{ It has been scientifically proven that mental exercise can prevent a person from getting } \\
\hline Alzheimer's disease $(\mathrm{F})$ & 20 & $0.20 \pm 0.4$ \\
\hline People in their 30s can have Alzheimer's disease (T) & 73.3 & $0.73 \pm 0.5$ \\
\hline Having high cholesterol may increase a person's risk of developing Alzheimer's disease (T) & 53.3 & $0.53 \pm 0.5$ \\
\hline Prescription drugs that prevent Alzheimer's disease are available (F) & 60 & $0.60 \pm 0.5$ \\
\hline \multicolumn{3}{|l|}{ Having high blood pressure may increase a person's risk of developing Alzheimer's } \\
\hline disease $(\mathrm{T})$ & 60 & $0.60 \pm 0.5$ \\
\hline Genes can only partially account for the development of Alzheimer's disease (T) & 80 & $0.80 \pm 0.4$ \\
\hline Mean score & & $3.47 \pm 0.9$ \\
\hline
\end{tabular}

Symptoms

Tremor or shaking of the hands or arms is a common symptom in people with Alzheimer's disease (F)

Trouble handling money or paying bills is a common early symptom of Alzheimer's disease (T)

One symptom that can occur with Alzheimer's disease is the belief that other people are stealing one's things ( $\mathrm{T}$ )

Most people with Alzheimer's disease remember recent events better than things that happened in the past $(\mathrm{F})$

86.7
$\mathbf{3 . 0 0} \pm \mathbf{0 . 8}$

Mean score

\section{Treatment and Management}

People whose Alzheimer's disease is not yet severe can benefit from psychotherapy for depression and anxiety (T)

Poor nutrition can make the symptoms of Alzheimer's disease worse (T)

$93.3 \quad 0.93 \pm 0.3$

When a person has Alzheimer's disease, the use of reminder notes is a crutch that can contribute to decline $(\mathrm{F})$

Alzheimer's disease cannot be cured (T)

Assessment and diagnosis

When a person with Alzheimer's disease becomes agitated, a medical examination might reveal other health problems that caused the agitation (T)

If trouble with memory and confused thinking appear suddenly, this is likely due to Alzheimer's disease (F)

Symptoms of severe depression can be mistaken for symptoms of Alzheimer's disease (T) Alzheimer's disease is one type of dementia (T)

Mean score

Care giving

People with Alzheimer's disease do best with simple instructions given one step at a time (T) 100

$33.3 \quad 0.33 \pm 0.5$

When people with Alzheimer's disease begin to have difficulty taking care of themselves, caregivers should take over right away (F) 
Table 1 (continued)

If a person with Alzheimer's disease becomes alert and agitated at night, a good strategy is to try to make sure that the person gets plenty of physical activity during the day (T) When people with Alzheimer's disease repeat the same question or story several times, it is helpful to remind them that they are repeating themselves (F)

Once people have Alzheimer's disease, they are no longer capable of making informed decisions about their own care (F)

Mean score

Course of the disease

After symptoms of Alzheimer's disease appear, the average life expectancy is 6-12 years (T)

In rare cases, people have recovered from Alzheimer's disease (F)

A person with Alzheimer's disease becomes increasingly likely to fall down as the disease gets worse $(\mathrm{T})$

Eventually, a person with Alzheimer's disease will need 24-h supervision (T)

Mean score

Overall mean knowledge score (out of 30)

\section{Correct}

answers, \%

Mean \pm SD

60

$0.60 \pm 0.5$

80

$0.80 \pm 0.4$

26.7

$0.27 \pm 0.5$

$3.20 \pm 1.0$

\section{3}

$0.33 \pm 0.5$

53.3

$0.53 \pm 0.5$

66.7

$0.67 \pm 0.5$

$0.67 \pm 0.5$

The number of respondents was 15. T, true; F, false.

\section{Knowledge}

The overall mean knowledge score achieved (19.5 out of 30) suggests that the participants have limited knowledge about dementia. These findings are consistent with those of Poreddi et al. [17], who reported a lack of knowledge among Indian nursing students about dementia (16.8 on the knowledge score), and those of Smyth et al. [24], who identified a lack of knowledge about dementia among Australian health care staff. A separate study, however, found that Indian doctors and nurses possessed a moderate level of knowledge about dementia [19]. These differences between nurses' and nursing students' levels of knowledge about dementia might be due to their respective levels of training and experience. Smyth et al. [24] argued that dementia training improves knowledge of the disorder, whereas younger professionals seem to score higher in knowledge than older professionals. Nevertheless, a lack of training can, according to Patel and Prince [25], lead to limited understanding and reinforced stigmas. By way of comparison, in a systematic review exploring nurses' knowledge of dementia, Evripidou et al. [26] reported that, in general, nurses lack knowledge of dementia care, but interventional studies suggest that their knowledge increases after attending training programs.

In our study, $60 \%$ of the participants agreed that people with dementia are particularly prone to depression and that symptoms of severe depression can be mistaken for symptoms of Alzheimer's disease (73.3\%). Considering the prevalence rate of depressive disorders in the elderly Indian population (i.e., 21.9\%) [27], this finding is fairly promising. However, depression is generally not perceived as an important health issue in India [28].

The majority of the participants in the presentstudy (93.3\%) answered that Alzheimer's disease cannot be cured. This finding is not consistent with that of Poreddi et al. [17], who found that only $55.7 \%$ of nursing students in India agreed that Alzheimer's disease could not be cured. These differences in knowledge between nursing staff and nursing students might be explained by the fact that more than half of the participants in our study $(66.7 \%)$ 
Table 2. Attitude toward Alzheimer's Disease and Related Dementias Scale

\begin{tabular}{|c|c|c|c|}
\hline & Agree & Disagree & Mean \pm SD \\
\hline \multicolumn{4}{|l|}{ Social comfort } \\
\hline 1. It is rewarding to work with people who have ADRD. & 93.3 & 6.7 & $5.9 \pm 1.3$ \\
\hline 2. I am afraid of people with ADRD.* & 6.7 & 93.3 & $6.1 \pm 1.3$ \\
\hline 4. I feel confident around people with ADRD. & 60 & 40 & $4.8 \pm 2.2$ \\
\hline 5. I am comfortable touching people with ADRD. & 86.7 & 13.3 & $5.9 \pm 1.5$ \\
\hline 6. I feel uncomfortable being around people with ADRD.* & 33.3 & 66.7 & $4.9 \pm 2.1$ \\
\hline 8. I am not very familiar with ADRD.* & 53.3 & 46.7 & $4.1 \pm 2.1$ \\
\hline 9. I would avoid an agitated person with ADRD.* & 26.7 & 73.3 & $5.7 \pm 1.9$ \\
\hline 13. I feel relaxed around people with ADRD. & 53.3 & 46.7 & $4.3 \pm 2.3$ \\
\hline 16. I feel frustrated because I do not know how to help people with ADRD.* & 53.3 & 46.7 & $4.1 \pm 2.2$ \\
\hline 17. I cannot imagine caring for someone with ADRD.* & 40 & 60 & $4.6 \pm 2.2$ \\
\hline Mean score & & & $50.3 \pm 9.2$ \\
\hline \multicolumn{4}{|l|}{ Dementia knowledge } \\
\hline 3. People with ADRD can be creative. & 73.3 & 26.7 & $5.4 \pm 1.9$ \\
\hline 7. Every person with ADRD has different needs. & 93.3 & 6.7 & $6.3 \pm 1.3$ \\
\hline 10. People with ADRD like having familiar things nearby. & 93.3 & 6.7 & $6.5 \pm 1.1$ \\
\hline 11. It is important to know the history of people with ADRD. & 93.3 & 6.7 & $6.3 \pm 1.1$ \\
\hline 12. It is possible to enjoy interacting with people with ADRD. & 66.7 & 33.3 & $5.1 \pm 2.0$ \\
\hline 14. People with ADRD can enjoy life. & 60 & 40 & $4.9 \pm 2.2$ \\
\hline 15. People with ADRD can feel when others are kind to them. & 100 & & $6.5 \pm 0.5$ \\
\hline 18. I admire the coping skills of people with ADRD. & 80 & 20 & $5.0 \pm 1.6$ \\
\hline 19. We can do a lot now to improve the lives of people with ADRD. & 73.3 & 26.7 & $6.3 \pm 1.3$ \\
\hline 20. Difficult behaviors may be a form of communication for people with ADRD. & 73.3 & 26.7 & $5.2 \pm 1.9$ \\
\hline Mean score & & & $57.6 \pm 5.4$ \\
\hline Overall attitude score & & & $107 \pm 10.9$ \\
\hline
\end{tabular}

ADRD, Alzheimer's disease and related dementias. * Reverse scored item.

were nurses, and they had an average of 7.7 years of experience in caring for the elderly. About half $(53.3 \%)$ of the participants in our study also said that people with dementia could recover from the condition in rare cases. Even though this shows a lack of knowledge, the result is consistent with what Poreddi et al. [17] found among the nursing students they studied. The finding is surprising considering that most participants (93.3\%) stated that Alzheimer's disease could not be cured. It is possible that some participants were unaware that Alzheimer's disease is one of many other brain disorders that can also cause dementia.

Only $33.3 \%$ of the participants answered that the average life expectancy of patients is 6-12 years after dementia symptoms appear; this is consistent with the findings of the study of Poreddi et al. [17], in which $44.3 \%$ of the nursing students answered the same, indicating that both nurses and nursing students in India have poor knowledge about the life expectancy of people with dementia. However, it is unclear whether they think that a diagnosis of dementia is connected to life expectancy at all or they consider life expectancy to be either shorter than 6 years or longer than 12 years.

The lack of knowledge about dementia reported in our study could be explained by the fact that the nurses surveyed were relatively older and therefore educated before there was much focus on dementia in nurses' education. 


\section{Attitudes}

The overall mean score determining participants' attitudes toward dementia (i.e., $107 \pm$ 10.9) indicates that the nursing staffs have overall positive attitudes toward people living with dementia. This finding is consistent with the study of Poreddi et al. [17], who reported an overall mean score for dementia attitude of $95 \pm 1.5$ among nursing students.

The majority of the participants $(93.3 \%)$ in our study found it rewarding to work with people with dementia. On the other hand, less than half of the participants (46.7\%) felt familiar with Alzheimer as a disease. This result might be informed by their experiences of frustration due to not knowing how to help people with dementia (53.3\%). In comparison, Poreddi et al. [17] found that $69.9 \%$ of nursing students found it rewarding to work with people with dementia, even though as many as $71.3 \%$ expressed unfamiliarity in working with this group of patients. Similarly positive attitudes have been identified in other studies on nurses and care staff $[26,29,30]$.

The importance of maintaining a positive attitude toward people with dementia to promote the patient's psychological well-being is emphasized by Norbergh et al. [31]. In the present study, the majority of the participants (73.3\%) agreed that a lot can be done to improve the lives of people with dementia. However, only $60 \%$ agreed that a person living with dementia could enjoy life. The fact that as many as $40 \%$ of the participants believe that people with dementia are unable to enjoy life can be of concern since studies have shown that persons with dementia are able to communicate their feelings and thoughts about their lives and can name several factors that impact the quality of their lives [32].

In the present study, $73.3 \%$ of the participants agreed that the persons challenging behaviors may be forms of communication, which is consistent with the nursing students' answers in the study by Poreddi et al. [17] in which $60.7 \%$ of the subjects agreed with this statement as well. These findings are promising since it is known that certain behaviors can be a result of increased difficulties in communicating needs [33] and that behaviors might also be connected to pain, discomfort, a lack of social contact, or imbalanced levels of stimulation [34]. In other words, several factors, both biological and psychological, can interact to influence these behaviors.

Despite promising results from our study identifying an overall positive attitude toward people with dementia, the lack of knowledge reported might still influence the quality of care delivered.

Studies indicate that nursing students perceive limited learning outcomes because they have not observed or experienced systematic person-centered approaches in caring for people with dementia [35]. Nevertheless, having an adequate level of knowledge about dementia can lead to earlier diagnosis of the disease [36] and give persons with dementia more choices regarding their care and lower the stigma against them [22].

The overall findings of the present study emphasize the urgent need to improve knowledge about dementia in India. This need is supported by Sinha et al. [7], who argue that nursing staff should be trained to be knowledgeable and competent in providing institutional care, and undergraduate medical and nursing students' curricula should address geriatric health issues, including dementia.

\section{Strengths and Limitations}

The present study has some limitations that should be acknowledged, including its small sample size. Despite the small size, the participants included were from 3 different NH in different parts of India, and, to our knowledge, this is the first study to describe the knowledge and attitudes toward dementia among nursing staff in India. Further, the present sample only includes women, which made it impossible to compare possible gender differences, but, ultimately, the majority of nursing staff in Indian NH are women. 
Another limitation could be identified in the fact that the ADKS is not an assessment tool used in clinical nursing practice; rather, it is used in applied and research contexts to assess knowledge of Alzheimer's disease among laypeople, patients, caregivers, and professionals [21]. Therefore, the ADKS might exhibit a ceiling effect in different groups, such as nursing students, nurses, psychologists, and doctors.

\section{Conclusion}

These findings reveal that nursing staff have limited knowledge of dementia, but their attitudes toward people living with dementia are positive. These results confirm the need to improve knowledge about dementia among nursing staff in India to enable them to provide high-quality care for $\mathrm{NH}$ residents with dementia. We therefore suggest extending this line of research to a larger sample in order to improve nursing staff's knowledge and attitudes surrounding dementia in India.

\section{Acknowledgement}

We would like to express our gratitude to Helene Kolnes, Maren Rogneby, Ingrid J. Brevik, Solveig Skåland, Amalie Tangen, Anne-Marie Landeg, Malene Berg, Marthine Skogfelt, and Silje J. Horn, all nursing students from VID Specialized University in Oslo, Norway, for their contributions to this study in collecting data.

\section{Statement of Ethics}

This study was accepted by the Norwegian social science data services (NSD). As no residents were interviewed or examined, ethical approval was not required, in accordance with the Norwegian Act on Medical and Health Research and confirmed by the NSD (reference No. 57526).

\section{Disclosure Statement}

The authors declare no conflict of interests.

\section{References}

1 Prince M, Wimo A, Guerchet MM, Ali GC, Wu YT, Prina M. World Alzheimer report 2015: the Global impact of Dementia - an analysis of prevalence, incidence, cost and trends. London: Alzheimer's Disease International; 2015.

2 Shaji KS. Dementia care in developing countries: the road ahead. Indian J Psychiatry. 2009 Jan;51 Suppl 1:S5-7.

3 Gupta R. Systems perspective: understanding care giving of the elderly in India. Health Care Women Int. 2009 Dec;30(12):1040-54.

4 Ugargol AP, Hutter I, James KS, Bailey A. Care Needs and Caregivers: Associations and Effects of Living Arrangements on Caregiving to Older Adults in India. Ageing Int. 2016;41(2):193-213.

5 Chatterji S, Kowal P, Mathers C, Naidoo N, Verdes E, Smith JP, et al. The health of aging populations in China and India. Health Aff (Millwood). 2008 Jul-Aug;27(4):1052-63.

6 Shaji KS, Jithu VP, Jyothi KS. Indian research on aging and dementia. Indian J Psychiatry. 2010 Jan;52(7 Suppl 1):S148-52. 
7 Sinha P, Das P, Sharma N, Bhattacharjee S. A case of Hansen's disease presenting with sulfone syndrome and hemolytic anemia. Indian J Public Health. 2011;55(2):67-9.

8 Rao GN, Bharath S. Cost of dementia care in India: delusion or reality? Indian J Public Health. 2013 Apr-Jun; $57(2): 71-7$

9 Alphonso T, Krishnamoorthy ES, Gomes K. Caregiving for dementia: global perspective and transcultural issues. In: Ennapadam S, Krishnamoorthy MJP, Cummings JL, editor. Dementia: a global approach. 1st ed. New York: Cambridge University Press; 2010.

10 Shaji KS, Smitha K, Lal KP, Prince MJ. Caregivers of people with Alzheimer's disease: a qualitative study from the Indian 10/66 Dementia Research Network. Int J Geriatr Psychiatry. 2003 Jan;18(1):1-6.

11 Kang Y, Moyle W, Venturato L. Korean nurses' attitudes towards older people with dementia in acute care settings. Int J Older People Nurs. 2011 Jun;6(2):143-52.

12 Alzheimer's and Related Disorders Society of India. The Dementia India Report: prevalence, impact, costs and services for Dementia. New Delhi: ARDSI; 2010.

13 Moyle W, Borbasi S, Wallis M, Olorenshaw R, Gracia N. Acute care management of older people with dementia: a qualitative perspective. J Clin Nurs. 2011 Feb;20(3-4):420-8.

14 Robinson A, Cubit K. Caring for older people with dementia in residential care: nursing students' experiences. J Adv Nurs. 2007 Aug;59(3):255-63.

15 Saini R, Alagh P, Carpenter B. Nurses and Alzheimer's disease: a holistic perspective. Indian J Public Health. 2012 Oct-Dec;56(4):318-9.

16 Kaur S, Kumar A, Kaur B, Rani B, Ghai S. Knowledge and attitude regarding care of elderly among nursing students: an Indian perspective. J Nurs Care. 2014;3(3).

17 Poreddi V, Carpenter BD, Gandhi S, Chandra R, GandhiSuresh BadaMath S. Knowledge and attitudes of undergraduate nursing students toward dementia: an Indian perspective. Invest Educ Enferm. 2015 Dec;33(3): 519-28.

18 Prince M; 10/66 Dementia Research Group. Care arrangements for people with dementia in developing countries. Int J Geriatr Psychiatry. 2004 Feb;19(2):170-7.

19 Biswas A, Shantanu D, Sujit S, Uttam M, Manabendra M, Shyamal KD. Knowledge about dementia among doctor and nurses in a medical college hospital in India. J Alzheimer's Dis Parkinsonism. 2017;2(1):1-9.

20 Spector A, Revolta C, Orrell M. The impact of staff training on staff outcomes in dementia care: a systematic review. Int J Geriatr Psychiatry. 2016 Nov;31(11):1172-87.

21 Carpenter BD, Balsis S, Otilingam PG, Hanson PK, Gatz M. The Alzheimer's Disease Knowledge Scale: development and psychometric properties. Gerontologist. 2009 Apr;49(2):236-47.

22 Spector A, Orrell M, Schepers A, Shanahan N. A systematic review of 'knowledge of dementia' outcome measures. Ageing Res Rev. 2012 Jan;11(1):67-77.

23 O'Connor ML, McFadden SH. Development and psychometric validation of the Dementia Attitudes Scale. Int J Alzheimers Dis. 2010;2010:1-10.

24 Smyth W, Fielding E, Beattie E, Gardner A, Moyle W, Franklin S, et al. A survey-based study of knowledge of Alzheimer's disease among health care staff. BMC Geriatr. 2013 Jan;13(1):2.

25 Patel V, Prince M. Ageing and mental health in a developing country: who cares? Qualitative studies from Goa, India. Psychol Med. 2001 Jan;31(1):29-38.

26 Evripidou M, Charalambous A, Middleton N, Papastavrou E. Nurses' knowledge and attitudes about dementia care: systematic literature review. Perspect Psychiatr Care. 2019 Jan;55(1):48-60.

27 Barua A, Ghosh M, Kar N, Basilio M. Distribution of depressive disorders in the elderly. J Neurosci Rural Pract. 2010 Jul;1(2):67-73.

28 Satcher DS. Executive summary: a report of the Surgeon General on mental health. Public Health Rep. 2000; 115(1):89-101.

29 Kim JH. Effects of gerontological nursing practicum on attitudes toward elders with dementia and general elders among Korean nursing students. Taehan Kanho Hakhoe Chi. 2006 Jun;36(4):645-51.

30 Moyle W, Murfield JE, Griffiths SG, Venturato L. Care staff attitudes and experiences of working with older people with dementia. Australas J Ageing. 2011 Dec;30(4):186-90.

31 Norbergh KG, Helin Y, Dahl A, Hellzén O, Asplund K. Nurses' attitudes towards people with dementia: the semantic differential technique. Nurs Ethics. 2006 May;13(3):264-74.

32 Mjørud M, Engedal K, Røsvik J, Kirkevold M. Living with dementia in a nursing home, as described by persons with dementia: a phenomenological hermeneutic study. BMC Health Serv Res. 2017 Jan;17(1):93.

33 Hancock GA, Woods B, Challis D, Orrell M. The needs of older people with dementia in residential care. Int J Geriatr Psychiatry. 2006 Jan;21(1):43-9.

34 Cohen-Mansfield J. Nonpharmacologic interventions for inappropriate behaviors in dementia: a review, summary, and critique. Am J Geriatr Psychiatry. 2001;9(4):361-81.

35 Skaalvik MW, Normann HK, Henriksen N. Student experiences in learning person-centred care of patients with Alzheimer's disease as perceived by nursing students and supervising nurses. J Clin Nurs. 2010 Sep;19(1718):2639-48.

36 Millard FB, Kennedy RL, Baune BT. Dementia: opportunities for risk reduction and early detection in general practice. Aust J Prim Health. 2011;17(1):89-94. 